\title{
Fabrication and Characterization of Ta-GelMA-BG Scaffolds by Chemical Crosslinking Processing for Promotion Osteointegration
}

\author{
Fujian $\mathrm{Zhao}^{1 \dagger}$, Xiongfa $\mathrm{Ji}^{2 \dagger}$, Yang Yan ${ }^{3,4}$, Zhen Yang ${ }^{3,4}$, Xiaofeng Chen ${ }^{3,4}$ and Limin $\mathrm{Ma}^{2 *}$ \\ ${ }^{1}$ Stomatological Hospital, Southern Medical University, Guangzhou, China, ${ }^{2}$ Department of Orthopedics, Guangdong Provincial \\ People's Hospital, Guangdong Academy of Medical Sciences, Guangzhou, China, ${ }^{3}$ Department of Biomedical Engineering, \\ School of Materials Science and Engineering, South China University of Technology, Guangzhou, China, ${ }^{4}$ National Engineering \\ Research Center for Tissue Restoration and Reconstruction, South China University of Technology, Guangzhou, China
}

The repair of bone defects in load-bearing positions still faces great challenges. Tantalum (Ta) has attempted to repair bone defects based on the excellent mechanical properties. However, the osseointegration of Ta needs to be improved due to the lack of osteoinduction. Herein, tantalum-gelatin-methacryloyl-bioactive glass (Ta-GelMA-BG) scaffolds were successfully fabricated by loading BG in 3D-printed Ta scaffolds through a chemical crosslinking method. The results showed that the composite scaffolds have the ability to promote cell adhesion and proliferation. The incorporation of $B G$ resulted in a significant increase in apatite-forming and osteogenesis differentiation abilities. In vivo results indicated that the Ta-GeIMA-BG scaffolds significantly enhanced the osteointegration at the early stage after implantation. Overall, the Ta-GelMA-BG scaffolds are a promising platform for the load bearing bone regeneration field.

Keywords: tantalum, bioactive glass, osteointegration, chemical crosslinking, bone tissue engineering

\section{INTRODUCTION}

Bone defects and nonunions caused by trauma, resection, and abnormal development pose a major clinical problem worldwide (Panteli et al., 2015). Current approaches for graft materials include autografts, allografts, ceramic, and metallic implants (Khan et al., 2008). The autografts or allografts have limited sources and are expensive (Greenwald et al., 2001). Ceramic implants cannot support the body weight and are only used with external fixation. Stress shielding often inevitably occurs and extraction surgery is needed to remove the fixation (Sumner, 2015). Metallic implants are suitable for the load-bearing bone defects. For example, the Ti- $6 \mathrm{Al}-4 \mathrm{~V}$ metal is still the most widely used material for orthopedics surgery (Thijs et al., 2010). However, concerns have also been raised for their unsuitable mechanical properties (Sagomonyants et al., 2011b), cytotoxicity (Li et al., 2010), corrosion, and potential allergies (Olmedo et al., 2008). These limitations of titanium have led to continuous efforts to explore more suitable metallic implant materials.

Tantalum $(\mathrm{Ta})$ has been shown to be a promising material for orthopedic clinical applications (Balla et al., 2010). Ta is a ductile, highly chemically resistant, biocompatible material (Black, 1994). However, the high price and difficulties in machining limit their clinical usage. Bulk Ta implants have high mechanical strength, and well-designed porous Ta has mechanical properties similar to those of human cancellous bone. This is vital for preventing the stress shielding (Arabnejad et al., 2017). Porous $\mathrm{Ta}$ favors the attachment and proliferation of osteoblasts as well as osteointegration (Stiehler 
et al., 2008; Sagomonyants et al., 2011a). More importantly, the porous structure can significantly reduce the weight and the cost of the implant (Lefebvre et al., 2008).

Traditional manufacturing techniques include furnace sintering, plasma spraying, lost wax casting, and vapor deposition techniques (Ryan et al., 2006). However, these techniques have difficulty satisfying the personalized demand for bone defects and precisely imitating trabecular structures. Different from traditional reduced material manufacturing, 3D printing is a bottom-to-top additive fabrication process. The low waste and high customized design endow it with a low material demand, which is suitable for the high price of the Ta metal (Wauthle et al., 2015). 3D printing solved the difficulties of machining the Ta metal, because the metal powder was used in layer-to-layer fabrication. For example, Wauthle et al. (2015) and Fraser et al. (2019) used selective laser melting (SLM) to manufacture a highly open porous $(80 \%)$ pure Ta implant. Within the limits of the cancellous bone, the porous Ta shows excellent mechanical properties, such as high elastic modulus and the ductile deformation mechanism. The satisfying bone ingrowth confirmed the success of the scaffold design (Endrizzi et al., 2016). However, since $\mathrm{Ta}$ is a chemically stable metal with no osteoinductivity, the bone in-growth rate is limited (Liu et al., 2015b). To meet the increasing requirements for earlier loading of implants in clinical applications, the surface modification of porous Ta is used to achieve faster bone ingrowth and more stable osseoincorporation (Roffi et al., 2017). Especially for the cases with large-volume bone defects, it is important for the porous tantalum to induce bone formation and strengthen the osseoincorporation (Candrian et al., 2017).

Bioactive glasses (BGs) have attracted increasing attention in recent years due to their osteoconductive and osteoinductive properties (Hench, 2016; Baino et al., 2018). Our previous work also proved that BG could successfully promote bone regeneration via immunomodulation (Zhao et al., 2018a; Zhao et al., 2018b). Moreover, BG is also used as a novel biocompatible degradable inorganic nanocarrier (Kim et al., 2016; Mahapatra et al., 2016; Kang et al., 2018; Patel et al., 2019). However, despite all of its advantages, it has fatal disadvantages. BG has low fracture toughness and cannot withstand rapid temperature changes, which leads to the formation of microcracks and then reduces the mechanical properties (Boccaccini et al., 2007). Therefore, although BG has been widely used in bone regeneration, it can only be used for the reconstruction of nonload-bearing bones (Jones, 2013). However, load-bearing capacity is especially important for bone scaffolds that are used to repair critical-sized bone defects in the load-bearing bones, because no loading or dramatically reduced loading on the bone scaffolds results in no stimulus for continued bone remodeling. According to Wolff s law, without proper loads placed under, no satisfactory bone remodeling will occur (Chen et al., 2010). Therefore, the bone scaffolds should be strong enough to bear loading for bone remodeling in the load-bearing sites.

This problem can be overcome by creating BG-Ta hybrid structures, which can be used as scaffolds to fill bone defects. The porous Ta scaffold is used to reconstruct the anatomy of the bone defect with mechanical stability and a sustained load at the site of the injury. Abundant porosities allow nutrients and oxygen to reach the osteoblasts seeded or migrated within. The surface modification of BG is designed to improve the osteoinductive and osteointegrative properties of the Ta scaffold. In order to attach BG to the Ta surface, a binder is necessary. Gelatin methacryloyl (GelMA) is an attractive chemically crosslinked hydrogel, which has excellent biocompatibility and biodegradability (Yue et al., 2015). To our knowledge, the combination of a 3D-printed Ta bionic structure and BG nanomolecules has not been explored in the field of load-bearing bone regeneration. Therefore, in this study, we use the SLM method to print the customized Ta scaffold. Surface chemical modification was used to photocrosslink with the BG-loaded GelMA. Both in vitro and in vivo experiments were carried out to evaluate the properties of the scaffolds and the osteoconductivity and osteoinductivity of the BG-GelMA coating.

\section{EXPERIMENTAL SECTION}

\section{Fabrication of Ta-GelMA-BG Scaffolds}

Fabrication of Ta scaffolds: Porous Ta scaffolds were fabricated via an SLM system (Concept Laser M2, Upper Franconia, Germany) with Unigraphics NX software (Siemens PLM Software, TX, United States). The printing process was protected in an $\mathrm{Ar} / \mathrm{N}_{2}$ atmosphere by using $\mathrm{Ta}$ powder particles (Siemens PLM Software, TX, United States) with the diameters in the range of $15-50 \mu \mathrm{m}$. The porous Ta samples were ultrasonically cleaned in ethyl alcohol and deionized water. The samples with diameters of $11 \mathrm{~mm}$ and $3 \mathrm{~mm}$ were used for in vitro and in vivo experiments, respectively.

Fabrication of GelMA: GelMA was synthesized following a previously reported method (Nichol et al., 2010). In brief, $10 \mathrm{~g}$ type A gelatin (Sigma, USA) was added to $100 \mathrm{ml}$ phosphate buffer saline (PBS) at $60^{\circ} \mathrm{C}$ until fully dissolved. $5^{\circ} \mathrm{ml}$ of methacrylic anhydride (Aladdin, China) was added dropwise into the solution at a rate of $0.5 \mathrm{ml} \mathrm{min}^{-1}$ under vigorous stirring for $3 \mathrm{~h}$. In order to remove the unreacted components, the mixture solution was dialyzed against deionized water for 1 week at $40^{\circ} \mathrm{C}$ using $12-14 \mathrm{kDa}$ cut-off dialysis tubes. After dialysis, the solution was centrifuged and filtered through a neutral filter paper. Finally, the GelMA solution was frozen at $-20^{\circ} \mathrm{C}$, lyophilized, and stored at $-80^{\circ} \mathrm{C}$.

Fabrication of Ta-GelMA-BG scaffolds: Ta-GelMA-BG scaffolds were prepared by adhering GelMA-BG sol on Ta scaffolds. The first step was to prepare the GelMA-BG sol. In brief, $\mathrm{BG} \quad\left(\mathrm{mol} \% \quad 60 \mathrm{SiO}_{2}-36 \mathrm{CaO}-4 \mathrm{P}_{2} \mathrm{O}_{5}\right)$ with a size of 400-500 nm was synthesized using the sol-gel method with alkali-catalyzed template as in our previously reported study (Zhao et al., 2018a). $1 \mathrm{~g}$ of lyophilized GelMA was dissolved into $10 \mathrm{ml}$ PBS containing a $0.5 \% \mathrm{w} / \mathrm{v}$ photoinitiator (Irgacure 2959, Aladdin, China) at 60 C. Subsequently, 10\% w/v BGs were dispersed into the GelMA solution and allowed to stir overnight at $40 \mathrm{C}$. Then, the Ta scaffolds were immersed into the solution and vacuumed for $30 \mathrm{~min}$. Subsequently, the Ta-GelMA-BG scaffolds were incubated at $4 \mathrm{C}$ for $30 \mathrm{~min}$ and immediately irradiated with UV light $(360-480 \mathrm{~nm})$ for $5 \mathrm{~min}$ at an 
intensity of $6.9 \mathrm{~mW} / \mathrm{cm}^{2}$. After crosslinking, the scaffolds were soaked in PBS overnight to remove the unreacted chemical components. Finally, the composite hydrogels were frozen at $-20^{\circ} \mathrm{C}$ for $12 \mathrm{~h}$ and lyophilized for $48 \mathrm{~h}$ to obtain interconnected porous hydrogels. The Ta-GelMA scaffolds were prepared using the same method, except without the addition of BG.

\section{Characterizations of Ta-GelMA-BG Scaffolds}

The surface morphology of Ta-GelMA-BG scaffolds was investigated through a field emission scanning electron microscope (SEM, DSM 982-Gemini or Sigma 300, Zeiss). The $\mathrm{X}$-ray diffraction analyzer (XRD, Bruker D8, Netherlands) at a scanning speed of $2 \%$ min and $2 \theta$ from 10 to $80^{\circ}$ was used to detect the phase composition of scaffolds. The porosities of the scaffolds were measured using the Archimedes' principle. The apatiteforming ability was investigated by immersing the scaffolds in SBF (VSBF/Mscaffolds: $50 \mathrm{ml} \mathrm{g-1)} \mathrm{for} 7$ days in a polyethylene bottle at $37 \mathrm{C}$. The scaffolds were collected from SBF after soaking, rinsed with distilled water 3 times, and dried at $60 \mathrm{C}$ overnight. Then, the apatite formation on the surface was analyzed by SEM.

\section{In Vitro Cytocompatibility and Osteogenesis Differentiation Studies}

Mouse bone marrow-derived mesenchymal stem cells (mBMSCs, ATCC) were used to study the cytocompatibility and osteogenesis differentiation properties of the scaffolds. The cells were seeded on the surface of scaffolds $(11 \mathrm{~mm}$ in diameter and $1.5 \mathrm{~mm}$ in thickness) at a density of $2 \times 104$ cells per sample and cultured with DMEM supplemented with $10 \%$ fetal bovine serum and $1 \%$ penicillin/streptomycin solution at $37 \mathrm{C}$ in $5 \% \mathrm{CO}_{2}$. After 2 days of culture, the cell attachment was analyzed by SEM observation.

The cell proliferation on scaffolds was investigated by a cell counting kit-8 (CCK-8, Dojindo, Japan). After 1, 3, and 7 days incubation, the scaffolds were rinsed with PBS, and then added with the fresh medium containing $10 \mathrm{v} / \mathrm{v} \%$ CCK-8 solution. After $1 \mathrm{~h}$ incubation, the absorbance of the fresh medium was recorded at a wavelength of $450 \mathrm{~nm}$ using a microplate reader (Thermo 3001, Thermo. Sci.).

Alizarin red-S assay was performed at day 14. In brief, after fixation with $4 \%$ paraformaldehyde for $30 \mathrm{~min}$, the cells were washed three times with ultrapure water and then submerged to a solution of 1\% Alizarin Red-S (pH 4.2, Sigma-Aldrich) for $10 \mathrm{~min}$ at room temperature.

The relative gene expression levels of osteogenic differentiation (OCN, OPN, RUNX2, COL1, and ALP) were analyzed by the real-time quantitative polymerase chain reaction (RT-qPCR) at 7 days. Typically, total RNA was extracted using HiPure Total RNA Micro Kit (Magen) following the manufacturer's instructions. The isolated RNA was then reverse transcribed into cDNA by using the Reverse Transcription Reagents Kit (Takara). The RT-qPCR was performed by using a Maxima SYBR Green/ROX qPCR
(Thermo Scientific) and conducted on a Quantstudio 6 Flex (Life technologies). The gene expressions were calculated by the $2^{-\Delta \Delta C t}$ method. The sequences of primers for OCN, OPN, RUNX2, COL1, and ALP genes were given in Supplementary Table S1. The relative expression of the genes was normalized against the housekeeping gene GAPDH.

\section{In Vivo Bone Regeneration Assays}

Surgical procedure and treatment: Adult female SpragueDawley rats (220-250 g) were provided by the Laboratory Animal Center, Southern Medical University. Before the surgical procedure, the rats were anesthetized with $10 \%$ chloral hydrate by intraperitoneal injection and the surgical areas were disinfected by iodine and ethyl alcohol. A femoral condyles defect model with a $3 \mathrm{~mm}$ diameter $\times 3 \mathrm{~mm}$ deep was formed on the femoral condyles by using an electric trephine drill. Then, Ta, Ta-GelMA, and Ta-GelMA-BG scaffolds were used to fill in the bone defects (Supplementary Figure S1). The wounds were sutured and a prophylactic antibiotic was administered to avoid infections.

Histological analysis: After 8 weeks, the rats were euthanized by an overdose of anesthetics and their femoral samples were harvested. Then the bone specimens were fixed in $4 \%$ paraformaldehyde, dehydrated in a series of ethanol concentrations, and embedded in polymethylmethacrylate (PMMA). Subsequently, they were initially processed into thicker sections $(150 \mu \mathrm{m})$ with a Leica SP1600 saw microtome (Leica, Hamburg, Germany), and were then sectioned into thinner sections $(\sim 50 \mu \mathrm{m})$ by grinding and polishing. To visualize the formed new bone, all the slices were stained with hematoxylin and eosin (H\&E), and were then imaged with a phase contrast light microscope to observe the new bone formation and osseointegration.

\section{Statistical Analysis}

Data were expressed as mean \pm standard deviation (SD). The statistical significances were determined via the Student's $t$-test or one-way analysis of variance (ANOVA) in SPSS 26.0 software (IBM SPSS Inc. Chicago, United States). The difference was considered to be statistically significant when $p<0.05$.

\section{RESULTS}

\section{Characterizations of the Ta-GelMA-BG Scaffolds}

In this study, Ta-GelMA-BG scaffolds were successfully prepared by adhering GelMA-BG sol on the Ta scaffold's surface, through an UV crosslinking process as shown in Figure 1A. Due to the attachment of BG, the color of Ta-GelMA-BG scaffolds was whiter than that of the $\mathrm{Ta}$ scaffolds, as shown in the digital photos (Figure 1B). The microstructure of the scaffolds was further observed by SEM and the average bore diameter of Ta scaffolds was approximately $400 \mathrm{~nm}$. Although the aperture of GelMA and GelMA-BG was reduced, the porous structure could still be observed. In addition, the BG particles had evenly dispersed in the GelMA gel. 

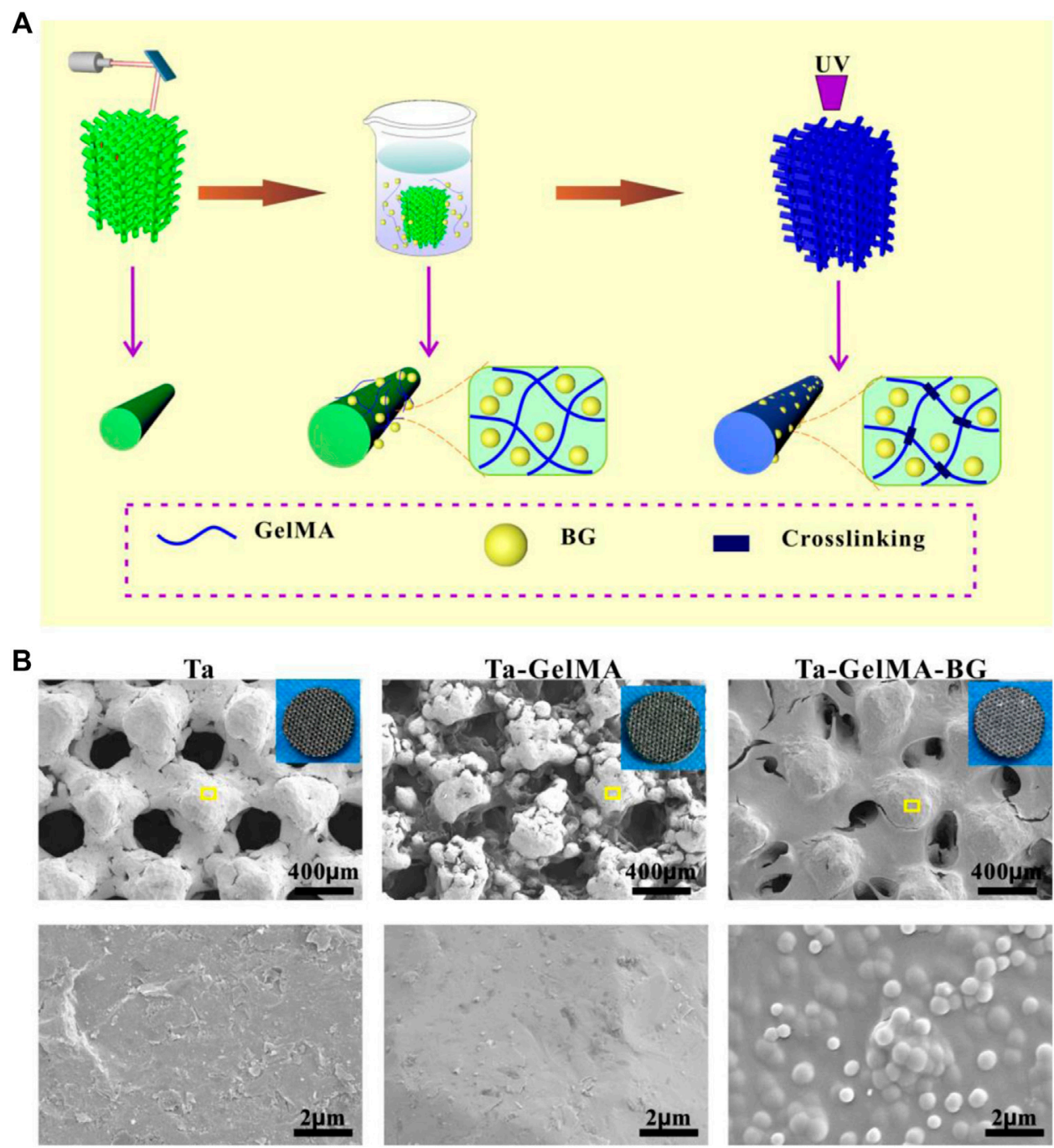

FIGURE 1 | Fabrication and observation of Ta-GelMA-BG scaffolds. (A) Schematic illustration of the fabrication process of Ta-GelMA-BG scaffolds. (B) SEM analysis of the surface structure of Ta, Ta-GelMA, and Ta-GelMA-BG scaffolds. The inserted image of B shows digital photos of the scaffolds and the yellow box indicates the enlarged area.

The porosity and mineralization properties of Ta-GelMA-BG scaffolds were further detected (Figure 2). The XRD analysis showed that the characteristic peaks of Ta were observed in all three groups. In addition, a broad peak centered at $\sim 22^{\circ}(2 \theta)$ could be observed only in Ta-GelMA-BG group indicating the representative amorphous nature of BG (Figure 2A). The porosity of the scaffolds was tested as shown in Figure 2B. Due to the adhesion of GelMA-BG, the porosity of Ta-GelMA-BG scaffolds showed a decreasing trend. Furthermore, the apatite formation was researched by incubating the scaffolds in SBF for $7^{\circ}$ days (Figure 2C), as the apatite-forming ability plays an important role in promoting osteointegration. There is no mineralization on the surface of $\mathrm{Ta}$ and $\mathrm{Ta}-\mathrm{GelMA}$, due to the lack of $\mathrm{Si}, \mathrm{Ca}$, and $\mathrm{P}$ ions from the SEM images results. In contrast, much BG remained on the surface of Ta-GelMA-BG scaffolds after $7^{\circ}$ days of degradation. Further enlargement showed that most of the BGs had changed into flake-like apatite. All of these results suggested that Ta-GelMA-BG scaffolds were successfully fabricated and had excellent porosity and mineralization properties.

\section{In vitro Biocompatibility and Osteogenesis of Ta-GelMA-BG Scaffolds}

The attachment and proliferation of mBMSCs on the surfaces of $\mathrm{Ta}, \mathrm{Ta}-\mathrm{GelMA}$, and Ta-GelMA-BG were examined by seeding the cells on the scaffolds. SEM images results indicated that all the groups were beneficial for cell attachment after culturing for $2^{\circ}$ days (Figure 3A). However, Ta group had significantly fewer cells than the other two groups indicating the only using Ta had a weak effect on promoting cell adhesion. Further observation showed that the prominent filopodia extension and spread areas of Ta-GelMA were better than Ta-GelMA-BG group, which might be due to the hydrophobic characteristics of $B G$ 


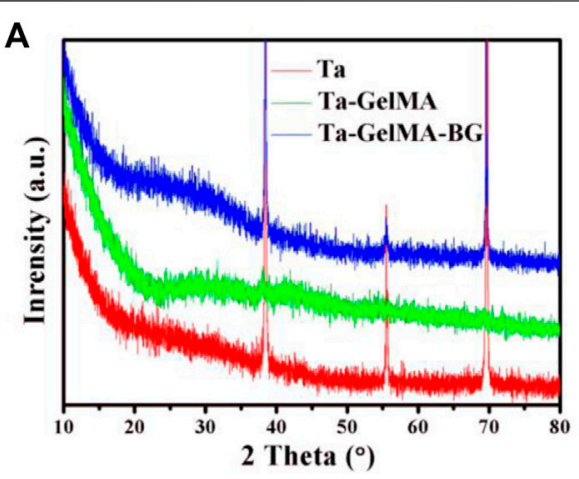

C
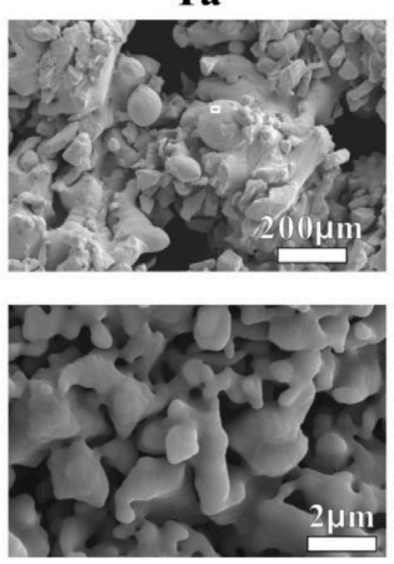

B

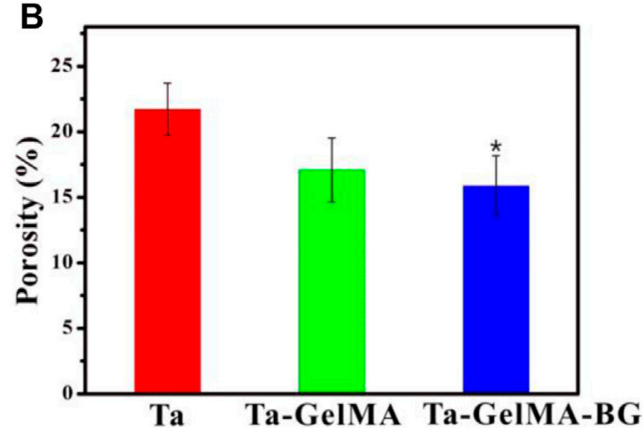

Ta-GelMA

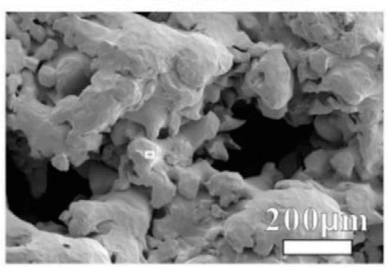

Ta-GelMA-BG
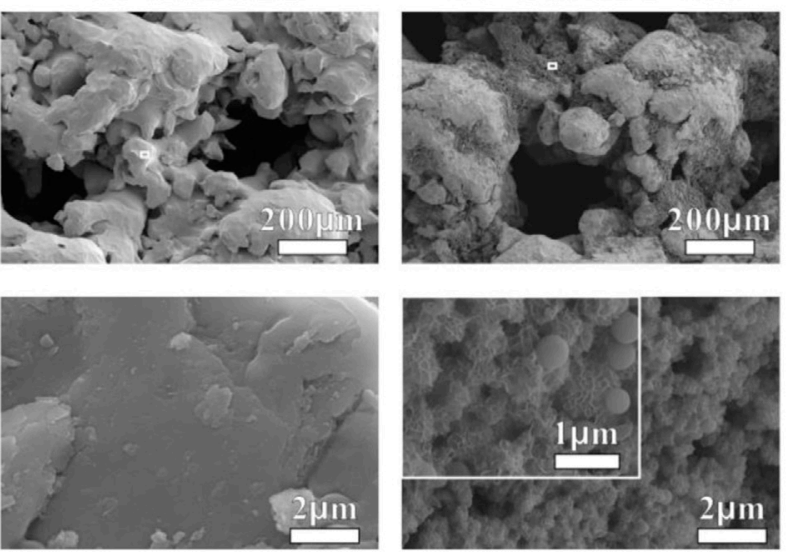

FIGURE 2 | Property evaluation of Ta-GelMA-BG scaffolds. (A) XRD pattern of the scaffolds. (B) Porosity of the scaffolds. (C) SEM images of the scaffolds after soaking in SBF for 7 days. $\left(^{*}\right) p<0.05$ vs Ta group.

were not suitable for cell growth. The CCK-8 results further verified the phenomenon of cell proliferation observed by SEM. There was no significant difference at 1 and $3^{\circ}$ days. However, when the culture time increased to $7^{\circ}$ days, the proliferation rates on Ta-GelMA-BG were significantly higher than those on $\mathrm{Ta}$ $(p<0.05)$ (Figure 3B).

The in vitro osteogenic differentiation of mBMSCs on the Ta, Ta-GelMA, and Ta-GelMA-BG scaffolds was also studied (Figure 3CD). The Alizarin red-S staining result indicated that all the three groups had the function of promoting mineralization. However, Ta-GelMA-BG was stained more deeply than the other two groups (Figure 3C). In addition, osteogenesis-related gene expression was also tested by RT-qPCR (Figure 3D). The OPN, RUNX2, COL1, and ALP expressions in the Ta-GelMA-BG group were significantly upregulated compared with those in the Ta and Ta-GelMA groups at day 7 . All of these results demonstrated that the Ta-GelMA-BG scaffolds could significantly promote the adhesion, proliferation, and osteogenic differentiation of mBMSCs.

\section{In vivo Osteointegration Assessment}

The in vivo osteointegration was evaluated by implanting Ta-GelMA-BG scaffolds in the rat femur bone for $8^{\circ}$ weeks (Supplementary Figure S1). The H\&E staining results showed that the scaffolds were surrounded by a lot of new bones in all the three groups (Figure 4). But the osteogenesis effect inside the scaffolds was significantly different. The Ta group was almost fully filled with fibrous connective tissues and cannot find a new bone. Similarly, very little new bones could be observed on the surface of the scaffold fibers of the Ta-GelMA group (yellow arrow in Figure 4). However, in the Ta-GelMA-BG group, a large amount of new bones had attached to the surface of the scaffold indicating the addition of BG significantly promoted osseointegration.

\section{DISCUSSION}

The promoting osteointegration of implanted biomaterials could significantly enhance bone regeneration (Agarwal and García, 2015). In this study, 3D printing was used to print the customized Ta scaffold with designed pore geometry; BG was used to improve the osteoinduction of the Ta scaffolds. We explored the effect of BG in Ta-GelMA-BG scaffolds on bone induction both in vitro and in vivo. The in vitro results showed that Ta-GelMA-BG can significantly promote the osteogenesis compared with $\mathrm{Ta}$ andTa-GelMA. In vivo experiments confirmed that Ta-GelMA-BG significantly promoted the osteointegration and new bone regeneration. 


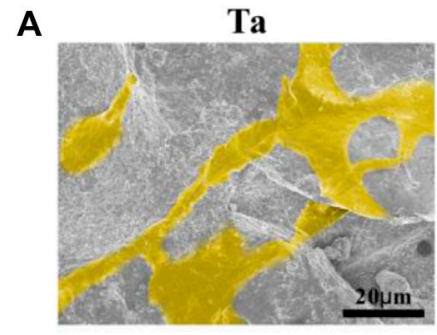

B

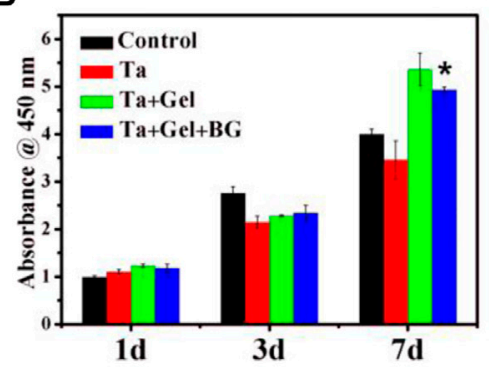

Ta-GelMA

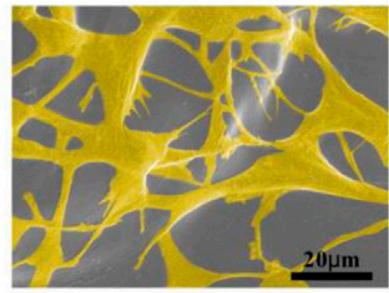

C

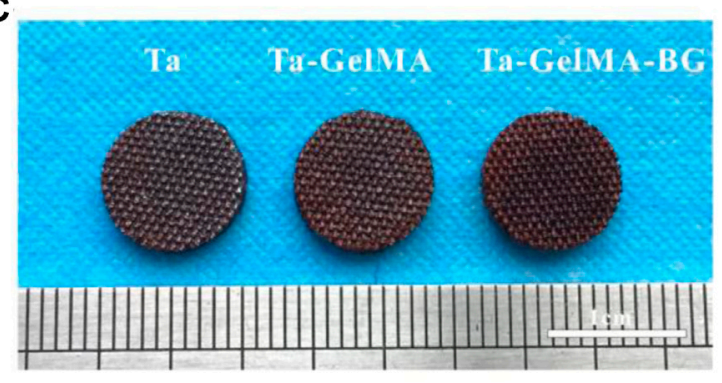

\section{D}

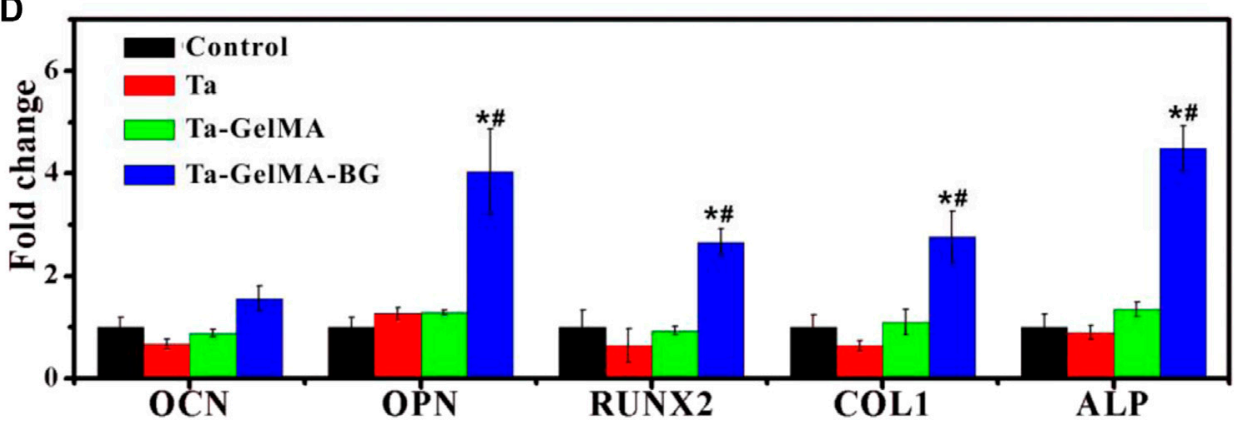

FIGURE 3 | In vitro biocompatibility and osteogenesis of Ta-GelMA-BG scaffolds: (A) SEM images of mBMSCs' attachment and spreading at day 2; (B) CCK-8 assay indicating the cell proliferation at day 1, 3, and 7; (C) Alizarin red of mBMSCs cultured with the scaffolds at 14 days; (D) mRNA expression of osteogenesis-related genes (OCN, OPN, RUNX2, COL1, and ALP) incubated with various groups for 7 days. $\left(^{*}\right) p<0.05$ vs Ta group; $\left({ }^{\#}\right) p<0.05$ vs Ta-GelMA group.

It is well-known that the difficulties of machining and the high cost of the Ta materials limit their application in the clinic (Ngo et al., 2018). Numerous researches have used additive manufacturing to build up metallic implants (Qin et al., 2019). The ready-to-use fabrication and highly customized design make this technique suitable for the complicated bone defect. In our study, SLM-based Ta scaffolds benefited from less material consumption, because of the layer-to-layer fabrication and the reusable unsintered metallic powder. For load-bearing applications, the proper pore design of the thin layer on the top of a solid substrate is required for the fast and solid anchoring of the implant (Shao et al., 2017). Compared to the identical Ta porous structures, our SLM-produced Ta is fabricated according to the optimization of geometric and mechanical properties for optimal load transfer and bone ingrowth. The pore design of Ta not only changed the elastic modulus, but also eased the intraoperative manipulation of the implant, which could obtain an optimal implant-bone fit and match the cancellous bone better.

In previous reports, the porous Ta implant has been widely used in clinical application with excellent osteointegration (Jenkins et al.,
2017). However, the highly stable chemical properties of the tantalum metal may delay its bonding to the bone tissue and bone ingrowth into porous structures (Arabnejad et al., 2016), because the lack of osteoinductivity of Ta determines the bone ingrowth starting from the margin of the scaffold. In addition, when the bone defect is large enough, the ingrowth bone around may inhibit the vasculature from reaching the center of the scaffold, thus the nutrition supply is insufficient for the bone regeneration (Luo et al., 2015). In this case, the early osteoinduction is important for the successful bone healing of the large-volume bone defect (García-Gareta et al., 2015). To meet the requirements, osteoinductive surface modification of the Ta scaffold is important for the successful bone regeneration in the large defect.

There are many ways to modify the surface characteristics of the Ta metal to optimize osteoinduction in vivo, including calcium phosphate coating (Barrère et al., 2003), alkaline-heat treatment (Miyazaki et al., 2000), anodic oxidation (Liu et al., 2015a; Liu et al., 2015b), and surface modification (Shah et al., 2019). Among these methods, chemically stable compounds are more suitable for the clinical translation. In our previous work, 

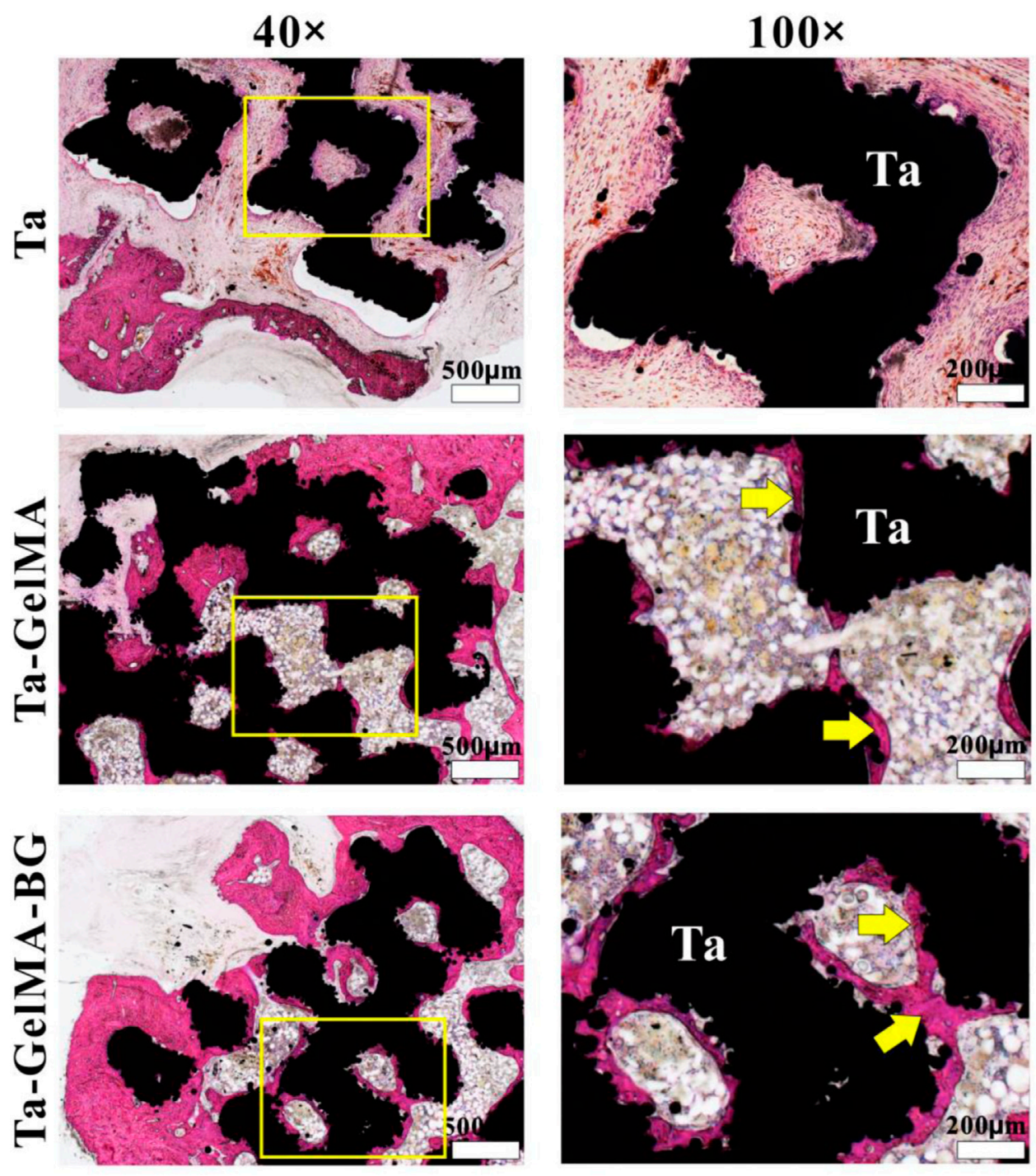

FIGURE 4 | In vivo osteointegration after implantation in femurs defect for 8 weeks by H\&E staining. The yellow arrows indicate the newly formed bone.

mineralized collagen, made of hydroxyapatite (HAp) nanocrystals and collagen fibers, was combined with 3Dprinted porous Ti6Al4V scaffold to improve the properties of vascularization, osteointegration, and osteogenesis (Ma et al., 2018). Numerous in vivo studies have also showed that bioceramic/bioglass scaffolds promote bone formation (Huang et al., 2020). In this study, our experiments showed that the successful loading of BG in the GelMA coating on the surface of Ta scaffold. The significantly improved osteogenesis is consistent with the previous reports of the surface modification of BG.

The possible mechanism of promoting early osteogenesis for Ta-GelMA-BG is that after Ta-GelMA-BG was implanted into the femoral condyle, $\mathrm{Si}, \mathrm{Ca}$, and $\mathrm{P}$ are quickly released from the GelMA coating. The $\mathrm{Si}, \mathrm{Ca}$, and $\mathrm{P}$ ions have the effect to directly stimulate osteogenesis. Meanwhile, the improved cell adhesion and proliferation benefit the osteointegration. Due to the excellent early osteoinduction ability of Ta-GelMA-BG in vtro and in vivo, the significantly improved bone regeneration in vivo was observed. Further studies should be carried out to investigate the molecular mechanism of BG-related host response for the osteogenesis and vascularization, as well as the mechanism of influence of directional mechanical stimulation on bone reconstruction.

\section{CONCLUSION}

In summary, we successfully fabricated the 3D-printed Ta-GelMA-BG scaffold by loading BG in Ta scaffolds through a chemical crosslinking approach. In vitro research found that the scaffolds had the ability to promote cell adhesion and proliferation. In addition, the incorporation of BG significantly promoted osteogenesis differentiation abilities. In vivo results showed that the Ta-GelMA-BG scaffolds had significantly enhanced the osteointegration during the early stage 
after implantation. Thus, Ta-GelMA-BG scaffold is a promising platform for the bone regeneration field.

\section{DATA AVAILABILITY STATEMENT}

The original contributions presented in the study are included in the article/Supplementary Material; further inquiries can be directed to the corresponding author.

\section{ETHICS STATEMENT}

The animal study was reviewed and approved by Guangdong Pharmaceutical University.

\section{AUTHOR CONTRIBUTIONS}

FZ and XJ wrote the manuscript; ZY, YY, and LM were involved in material preparation; $\mathrm{FZ}$ was involved in in vitro experiment;

\section{REFERENCES}

Agarwal, R., and García, A. J. (2015). Biomaterial Strategies for Engineering Implants for Enhanced Osseointegration and Bone Repair. Adv. Drug Deliv. Rev. 94, 53-62. doi:10.1016/j.addr.2015.03.013

Arabnejad, S., Burnett Johnston, R., Pura, J. A., Singh, B., Tanzer, M., and Pasini, D. (2016). High-strength Porous Biomaterials for Bone Replacement: A Strategy to Assess the Interplay between Cell Morphology, Mechanical Properties, Bone Ingrowth and Manufacturing Constraints. Acta Biomater. 30, 345-356. doi:10.1016/j.actbio.2015.10.048

Arabnejad, S., Johnston, B., Tanzer, M., and Pasini, D. (2017). Fully Porous 3D Printed Titanium Femoral Stem to Reduce Stress-Shielding Following Total Hip Arthroplasty. J. Orthop. Res. 35 (8), 1774-1783. doi:10.1002/jor.23445

Baino, F., Fiume, E., Miola, M., and Verné, E. (2018). Bioactive Sol-Gel Glasses: Processing, Properties, and Applications. Int. J. Appl. Ceram. Technol. 15 (4), 841-860. doi:10.1111/ijac.12873

Balla, V. K., Bose, S., Davies, N. M., and Bandyopadhyay, A. (2010). Tantalum-A Bioactive Metal for Implants. Jom 62 (7), 61-64. doi:10.1007/s11837-0100110-y

Barrère, F., van der Valk, C. M., Meijer, G., Dalmeijer, R. A. J., de Groot, K., and Layrolle, P. (2003). Osteointegration of Biomimetic Apatite Coating Applied onto Dense and Porous Metal Implants in Femurs of Goats. J. Biomed. Mater. Res. 67B (1), 655-665. doi:10.1002/jbm.b.10057

Black, J. (1994). Biologic Performance of Tantalum. Clin. Mater. 16 (3), 167-173. doi:10.1016/0267-6605(94)90113-9

Boccaccini, A. R., Chen, Q., Lefebvre, L., Gremillard, L., and Chevalier, J. (2007). Sintering, Crystallisation and Biodegradation Behaviour of Bioglass-Derived Glass-Ceramics. Faraday Discuss. 136, 27-44. doi:10.1039/b616539g

Chen, J.-H., Liu, C., You, L., and Simmons, C. A. (2010). Boning up on Wolff's Law: Mechanical Regulation of the Cells that Make and Maintain Bone. J. Biomech. 43 (1), 108-118. doi:10.1016/j.jbiomech.2009.09.016

Endrizzi, D. P., Mackenzie, J. A., and Henry, P. D. G. (2016). Early Debris Formation with a Porous Tantalum Glenoid Component. J. Bone Jt. Surg. 98 (12), 1023-1029. doi:10.2106/jbjs. 15.00410

Fraser, D., Mendonca, G., Sartori, E., Funkenbusch, P., Ercoli, C., and Meirelles, L. (2019). Bone Response to Porous Tantalum Implants in a gap-healing Model. Clin. Oral Impl Res. 30 (2), 156-168. doi:10.1111/clr.13402

García-Gareta, E., Coathup, M. J., and Blunn, G. W. (2015). Osteoinduction of Bone Grafting Materials for Bone Repair and Regeneration. Bone 81, 112-121. doi:10.1016/j.bone.2015.07.007
XC was involved in project administration, writing-review and editing; LM and XJ were involved in in vivo experiment; LM supervised the project. All authors read and approved the final manuscript.

\section{FUNDING}

This study was financially supported by the National Natural Science Foundation of China (Grant Nos. 32000933), the Natural Science Foundation of Guangdong Province (Grant Nos. 2019A1515110480, 2020A1515010827), and the Highlevel Hospital Construction Project (Grant Nos. KJ012019100).

\section{SUPPLEMENTARY MATERIAL}

The Supplementary Material for this article can be found online at: https://www.frontiersin.org/articles/10.3389/fmats.2021.701268/ full\#supplementary-material

Greenwald, A. S., Boden, S. D., Goldberg, V. M., Khan, Y., Laurencin, C. T., and Rosier, R. N. (2001). Bone-graft Substitutes: Facts, Fictions, and Applications The J. Bone Jt. Surgery-American Volume 83 (Suppl. 2 Pt 2), 98-103. doi:10.2106/00004623-200100022-00007

Hench, L. L. (2016). Bioglass: 10 Milestones from Concept to Commerce. J. NonCrystalline Sol. 432, 2-8. doi:10.1016/j.jnoncrysol.2014.12.038

Huang, Y., Li, L., Shi, Z., Cui, X., Pan, H., and Li, B. (2020). [Application and Research Status of Bioactive Glass in Bone Repair]. Zhongguo Xiu Fu Chong Jian Wai Ke Za Zhi 34 (5), 660-666. doi:10.7507/1002-1892.201908093

Jenkins, D. R., Odland, A. N., Sierra, R. J., Hanssen, A. D., and Lewallen, D. G. (2017). Minimum Five-Year Outcomes with Porous Tantalum Acetabular Cup and Augment Construct in Complex Revision Total Hip Arthroplasty. J. Bone Jt. Surg. 99 (10), e49. doi:10.2106/jbjs.16.00125

Jones, J. R. (2013). Review of Bioactive Glass: from Hench to Hybrids. Acta Biomater. 9 (1), 4457-4486. doi:10.1016/j.actbio.2012.08.023

Kang, M. S., Lee, N.-H., Singh, R. K., Mandakhbayar, N., Perez, R. A., Lee, J.-H., et al. (2018). Nanocements Produced from Mesoporous Bioactive Glass Nanoparticles. Biomaterials 162, 183-199. doi:10.1016/ j.biomaterials.2018.02.005

Khan, Y., Yaszemski, M. J., Mikos, A. G., and Laurencin, C. T. (2008). Tissue Engineering of Bone: Material and Matrix Considerations. J. Bone Jt. Surg Am 90 (Suppl. 1), 36-42. doi:10.2106/jbjs.g.01260

Kim, T.-H., Singh, R. K., Kang, M. S., Kim, J.-H., and Kim, H.-W. (2016). Inhibition of Osteoclastogenesis through siRNA Delivery with Tunable Mesoporous Bioactive Nanocarriers. Acta Biomater. 29, 352-364. doi:10.1016/ j.actbio.2015.09.035

Lefebvre, L.-P., Banhart, J., and Dunand, D. C. (2008). Porous Metals and Metallic Foams: Current Status and Recent Developments. Adv. Eng. Mater. 10 (9), 775-787. doi:10.1002/adem.200800241

Li, Y., Wong, C., Xiong, J., Hodgson, P., and Wen, C. (2010). Cytotoxicity of Titanium and Titanium Alloying Elements. J. Dent Res. 89 (5), 493-497. doi:10.1177/0022034510363675

Liu, Y., Bao, C., Wismeijer, D., and Wu, G. (2015a). The Physicochemical/ biological Properties of Porous Tantalum and the Potential Surface Modification Techniques to Improve its Clinical Application in Dental Implantology. Mater. Sci. Eng. C 49, 323-329. doi:10.1016/ j.msec.2015.01.007

Liu, Y., Bao, C., Wismeijer, D., and Wu, G. (2015b). The Physicochemical/ biological Properties of Porous Tantalum and the Potential Surface Modification Techniques to Improve its Clinical Application in Dental Implantology. Mater. Sci. Eng. C 49, 323-329. doi:10.1016/j.msec.2015.01.007 
Luo, Y., Zhai, D., Huan, Z., Zhu, H., Xia, L., Chang, J., et al. (2015). ThreeDimensional Printing of Hollow-Struts-Packed Bioceramic Scaffolds for Bone Regeneration. ACS Appl. Mater. Inter. 7 (43), 24377-24383. doi:10.1021/ acsami.5b08911

Ma, L., Wang, X., Zhao, N., Zhu, Y., Qiu, Z., Li, Q., et al. (2018). Integrating 3D Printing and Biomimetic Mineralization for Personalized Enhanced Osteogenesis, Angiogenesis, and Osteointegration. ACS Appl. Mater. Inter. 10 (49), 42146-42154. doi:10.1021/acsami.8b17495

Mahapatra, C., Singh, R. K., Kim, J.-J., Patel, K. D., Perez, R. A., Jang, J.-H., et al. (2016). Osteopromoting Reservoir of Stem Cells: Bioactive Mesoporous Nanocarrier/Collagen Gel through Slow-Releasing FGF18 and the Activated BMP Signaling. ACS Appl. Mater. Inter. 8 (41), 27573-27584. doi:10.1021/ acsami.6b09769

Miyazaki, T., Kim, H.-M., Miyaji, F., Kokubo, T., Kato, H., and Nakamura, T. (2000). Bioactive Tantalum Metal Prepared by $\mathrm{NaOH}$ Treatment. J. Biomed. Mater. Res. 50 (1), 35-42. doi:10.1002/(sici)1097-4636(200004)50:1<35:aidjbm6 $>3.0 . c 0 ; 2-8$

Ngo, T. D., Kashani, A., Imbalzano, G., Nguyen, K. T. Q., and Hui, D. (2018). Additive Manufacturing (3D Printing): A Review of Materials, Methods, Applications and Challenges. Composites B: Eng. 143, 172-196. doi:10.1016/ j.compositesb.2018.02.012

Nichol, J. W., Koshy, S. T., Bae, H., Hwang, C. M., Yamanlar, S., and Khademhosseini, A. (2010). Cell-laden Microengineered Gelatin Methacrylate Hydrogels. Biomaterials 31 (21), 5536-5544. doi:10.1016/ j.biomaterials.2010.03.064

Olmedo, D. G., Tasat, D. R., Evelson, P., Guglielmotti, M. B., and Cabrini, R. L. (2008). Biological Response of Tissues with Macrophagic Activity to Titanium Dioxide. J. Biomed. Mater. Res. 84A (4), 1087-1093. doi:10.1002/jbm.a.31514

Panteli, M., Pountos, I., Jones, E., and Giannoudis, P. V. (2015). Biological and Molecular Profile of Fracture Non-union Tissue: Current Insights. J. Cel. Mol. Med. 19 (4), 685-713. doi:10.1111/jcmm. 12532

Patel, K. D., Buitrago, J. O., Parthiban, S. P., Lee, J.-H., Singh, R. K., Knowles, J. C., et al. (2019). Combined Effects of Nanoroughness and Ions Produced by Electrodeposition of Mesoporous Bioglass Nanoparticle for Bone Regeneration. ACS Appl. Bio Mater. 2 (11), 5190-5203. doi:10.1021/ acsabm.9b00859

Qin, Y., Wen, P., Guo, H., Xia, D., Zheng, Y., Jauer, L., et al. (2019). Additive Manufacturing of Biodegradable Metals: Current Research Status and Future Perspectives. Acta Biomater. 98, 3-22. doi:10.1016/j.actbio.2019.04.046

Roffi, A., Krishnakumar, G. S., Gostynska, N., Kon, E., Candrian, C., and Filardo, G. (2017). The Role of Three-Dimensional Scaffolds in Treating Long Bone Defects: Evidence from Preclinical and Clinical Literature-A Systematic Review. Biomed. Res. Int. 2017 (2), 8074178. doi:10.1155/2017/ 8074178

Roffi, A., Krishnakumar, G. S., Gostynska, N., Kon, E., Candrian, C., and Filardo, G. (2017). The Role of Three-Dimensional Scaffolds in Treating Long Bone Defects: Evidence from Preclinical and Clinical Literature-A Systematic Review. Biomed. Res. Int. 2017, 1-13. doi:10.1155/2017/8074178

Ryan, G., Pandit, A., and Apatsidis, D. (2006). Fabrication Methods of Porous Metals for Use in Orthopaedic Applications. Biomaterials 27 (13), 2651-2670. doi:10.1016/j.biomaterials.2005.12.002

Sagomonyants, K. B., Hakim-Zargar, M., Jhaveri, A., Aronow, M. S., and Gronowicz, G. (2011a). Porous Tantalum Stimulates the Proliferation and Osteogenesis of Osteoblasts from Elderly Female Patients. J. Orthop. Res. 29 (4), 609-616. doi:10.1002/jor.21251
Sagomonyants, K. B., Hakim-Zargar, M., Jhaveri, A., Aronow, M. S., and Gronowicz, G. (2011b). Porous Tantalum Stimulates the Proliferation and Osteogenesis of Osteoblasts from Elderly Female Patients. J. Orthop. Res. 29 (4), 609-616. doi:10.1002/jor.21251

Shah, F. A., Thomsen, P., and Palmquist, A. (2019). Osseointegration and Current Interpretations of the Bone-Implant Interface. Acta Biomater. 84, 1-15. doi:10.1016/j.actbio.2018.11.018

Shao, H., Ke, X., Liu, A., Sun, M., He, Y., Yang, X., et al. (2017). Bone Regeneration in 3D Printing Bioactive Ceramic Scaffolds with Improved Tissue/material Interface Pore Architecture in Thin-wall Bone Defect. Biofabrication 9 (2), 025003. doi:10.1088/1758-5090/aa663c

Stiehler, M., Lind, M., Mygind, T., Baatrup, A., Dolatshahi-Pirouz, A., Li, H., et al. (2008). Morphology, Proliferation, and Osteogenic Differentiation of Mesenchymal Stem Cells Cultured on Titanium, Tantalum, and Chromium Surfaces. J. Biomed. Mater. Res. 86A (2), 448-458. doi:10.1002/jbm.a.31602

Sumner, D. R. (2015). Long-term Implant Fixation and Stress-Shielding in Total Hip Replacement. J. Biomech. 48 (5), 797-800. doi:10.1016/ j.jbiomech.2014.12.021

Thijs, L., Verhaeghe, F., Craeghs, T., Humbeeck, J. V., and Kruth, J.-P. (2010). A Study of the Microstructural Evolution during Selective Laser Melting of Ti6Al-4V. Acta Materialia 58 (9), 3303-3312. doi:10.1016/ j.actamat.2010.02.004

Wauthle, R., van der Stok, J., Amin Yavari, S., Van Humbeeck, J., Kruth, J.-P., Zadpoor, A. A., et al. (2015). Additively Manufactured Porous Tantalum Implants. Acta Biomater. 14, 217-225. doi:10.1016/j.actbio.2014.12.003

Yue, K., Trujillo-de Santiago, G., Alvarez, M. M., Tamayol, A., Annabi, N., and Khademhosseini, A. (2015). Synthesis, Properties, and Biomedical Applications of Gelatin Methacryloyl (GelMA) Hydrogels. Biomaterials 73, 254-271. doi:10.1016/j.biomaterials.2015.08.045

Zhao, F., Lei, B., Li, X., Mo, Y., Wang, R., Chen, D., et al. (2018a). Promoting In Vivo Early Angiogenesis with Sub-micrometer Strontium-Contained Bioactive Microspheres through Modulating Macrophage Phenotypes. Biomaterials 178, 36-47. doi:10.1016/j.biomaterials.2018.06.004

Zhao, F., Xie, W., Zhang, W., Fu, X., Gao, W., Lei, B., et al. (2018b). 3D Printing Nanoscale Bioactive Glass Scaffolds Enhance Osteoblast Migration and Extramembranous Osteogenesis through Stimulating Immunomodulation. Adv. Healthc. Mater. 7, 1800361. doi:10.1002/adhm.201800361

Conflict of Interest: The authors declare that the research was conducted in the absence of any commercial or financial relationships that could be construed as a potential conflict of interest.

Publisher's Note: All claims expressed in this article are solely those of the authors and do not necessarily represent those of their affiliated organizations, or those of the publisher, the editors and the reviewers. Any product that may be evaluated in this article, or claim that may be made by its manufacturer, is not guaranteed or endorsed by the publisher.

Copyright (c) 2021 Zhao, Ji, Yan, Yang, Chen and Ma. This is an open-access article distributed under the terms of the Creative Commons Attribution License (CC BY). The use, distribution or reproduction in other forums is permitted, provided the original author(s) and the copyright owner(s) are credited and that the original publication in this journal is cited, in accordance with accepted academic practice. No use, distribution or reproduction is permitted which does not comply with these terms. 